NASA Technical Memorandum 81876

NASA-TM-81876 19800024246

\title{
INTERLAMINAR STRESS SINGULARITIES AT A STRAIGHT FREE EDGE IN COMPOSITE LAMINATES
}

I, S, Raju and John H. Crews, Jr,

\section{FOR REFERENCE}

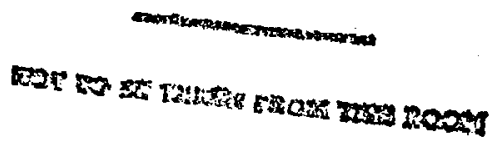

August 1980

\section{LEMARY Pis? \\ OCT 11980 \\ LATCLEY RTAEARCH CENTER \\ HMBRARY, UHASA

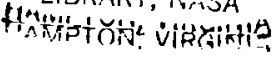




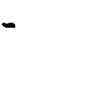




\title{
INTERLAMINAR STRESS SINGULARITIES AT A STRAIGHT \\ FREE EDGE IN COMPOSITE LAMINATES
}

\author{
I. S. Raju* and John H. Crews, Jr. \\ NASA Langley Research Center \\ Hampton, Virginia 23665
}

\section{SUMMARY}

A quasi-three-dimensional finite-element analysis was used to analyze the edge-stress problem in four-ply, composite laminates. The seven laminates that were considered belong to the laminate family $[\theta /(\theta-90)]$, where. $0 \leq \theta \leq 90$. Systematic convergence studies were made to explore the existence of stress singularities near the free edge. The present analysis appears to confirm the existence of stress singularities at the intersection of the interface and the free edge. The power of the stress singularity was the same for all seven laminates considered.

\section{INTRODUCTION}

Many composite laminates develop high interlaminar stresses near free edges due to mechanical loads, thermal loads, or hygroscopic effects. The interlaminar stresses can lead to delamination and eventual failure of the laminate. Several numerical analyses have computed stress distributions in a finite-width laminate subjected to uniform axial strain. This case is often referred to as the straight-edge or the edge-stress problem. In reference 1 , a finite-difference scheme was used to analyze the edge-stress problem of a $[ \pm 45]_{s}$ laminate. In reference 2, a three-dimensional finite-element method was used to analyze the edge effects in $[ \pm 45]_{s}$ and $[90 / 0]_{s}$ laminates. In reference 3 , a

\footnotetext{
*Joint Institute for Advancement of Flight Sciences, George Washington University.

This work was performed, in part, under NASA Grant NSG-1449.
} 
quas1-three-dimensional finite-element analysis was used to analyze [0/90] s' $[90 / 0]_{s},[ \pm 45]_{s}$, and several quasi-isotropic laminates. These analyses used coarse mesh models because of limitations on computing storages and times. However, all showed that the interlaminar stresses had very localized peaks near the free edge. If these localized peaks were caused by a singularity, as suggested in references 1 and 3 , singularity analyses could berdeveloped and used to study delaminations.

The present study investigated the existence of stress singularities at a free edge in several four-ply laminates that belong to the laminate family $[\theta /(\theta-90)]_{\text {s }}, \quad 0 \leq \theta \leq 90$. To this end, quasi-three-dimensional finite-element analysis was used. The analysis, which is similar to that of reference 3 , used elght-noded isoparametric elements.

The interlaminar stresses are presented as distributions through the thickness at the free edge, and along the interface between the plies. The results are compared and discussed for seven different laminates. Stress distributions calculated from the analyses were used in a procedure to investigate the existence of stress singularities.

\section{SYMBOLS}

\begin{tabular}{|c|c|}
\hline $\mathrm{b}$ & half-width of the straight-edge laminate, $m$ \\
\hline$E_{i i}$ & $\begin{array}{l}\text { Young's modulus in i-direction, MPa } \\
\ddots\end{array}$ \\
\hline$G_{i j}$ & shear modulus, $\mathrm{MPa}$ \\
\hline h & ply thickness, m \\
\hline r & nondimensional distance from the free edge, $r=(b-y) / h$ \\
\hline$, \mathrm{V}, \mathrm{W}$ & displacement functions, m \\
\hline
\end{tabular}




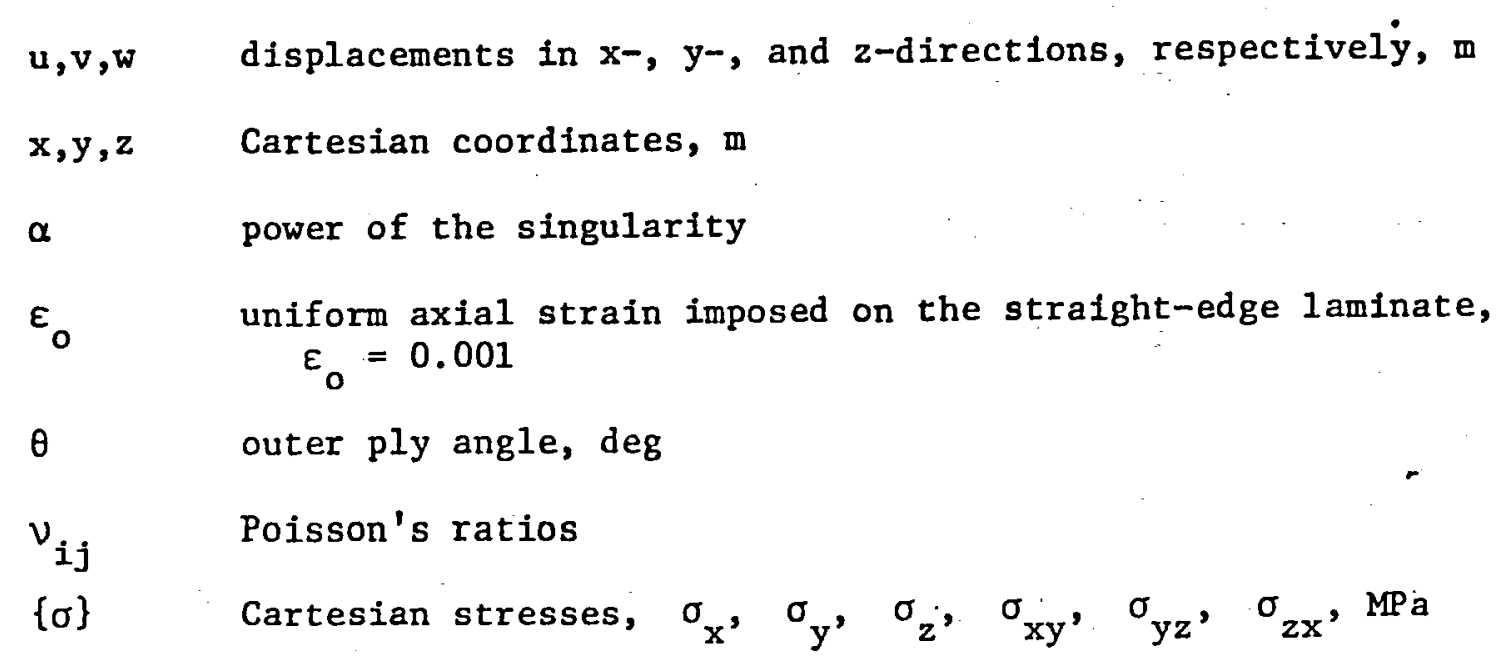

Subscripts:

$i, j \quad i, j=1,2,3$

1,2,3 longitudinal, transverse, and thickness directions of a unidirectional ply

\section{ANALYSIS}

This section describes the laminates analyzed, the quasi-three-dimensional formulation, and the finite-element idealizations used in this study. This section also discusses the procedure used to investigate the existence of a stress singularity,

\section{Laminates}

The laminates considered in the analyses were $[0 / 90] \mathrm{s},[15 /-75] \mathrm{s}$, $[30 /-60]_{s},[ \pm 45]_{s} ;[60 /-30]_{s},[75 /-15]_{s}$, and $[90 / 0]_{s} \cdot$ As previously mentioned, these seven laminates belong to the family $[\theta /(\theta-90)], 0 \leq \theta \leq 90$. The top ply angle, $\cdot \theta$, is shown in figure $I(a)$.

Each ply was idealized as a homogeneous, elastic orthotropic material with properties (refs. 1, 2, and 3), 


$$
\begin{aligned}
& E_{11}=137.9 \mathrm{GPa}\left(20 \times 10^{6} \mathrm{psi}\right) \\
& E_{22}=E_{33}=14.48 \mathrm{GPa}\left(2.1 \times 10^{6} \mathrm{psi}\right) \\
& G_{12}=G_{23}=G_{31}=5.86 \mathrm{GPa}\left(0.85 \times 10^{6} \mathrm{psi}\right) \\
& v_{12}=v_{13}=v_{23}=0.21
\end{aligned}
$$

The subscripts 1,2 , and 3 correspond to the longitudinal, transverse, and thickness directions, respectively, of the individual ply.

\section{Quasi-Three-Dimensional Analysis}

Figure $1(a)$ shows a long, symmetric laminate loaded in the $x$-direction. The laminate has a width of $2 b$ and has four plies, each of thickness $h$. Away from the ends, the displacements in any $x=$ constant plane were assumed to be

$$
\left.\begin{array}{l}
u(x, y, z)=\varepsilon_{0} x+v(y, z) \\
v(x, y, z)=v(y, z) \\
w(x, y, z)=w(y, z)
\end{array}\right\}
$$

where $\varepsilon_{0}$ is a uniform axial strain, and $U, V, W$ are functions of the coordinates $y$ and $z$ alone (ref. 1). Furthermore, because of symmetries inherent in the layups considered, $U, V$, and $W$ satisfy the following requiremențs:

$$
\left.\begin{array}{l}
U(y, z)=-U(-y,-z) \\
V(y, z)=-V(-y, z) \\
W(y, z)=-W(y,-z)
\end{array}\right\}
$$


and

$$
\frac{\partial}{\partial z} U(y, 0)=\frac{\partial}{\partial z} V(y, 0)=\frac{\partial}{\partial y} W(0, z)=0
$$

The displacement field of equations (1) should satisfy the equations of equilibrium and the stress-free conditions on the edges, $y= \pm b$, and top and bottom surfaces, $z= \pm 2 h$.

Because exact solutions are not available for the complex three-dimensional problem, approximate methods were used in references 1 : through 3 . The present study was based on a finite-element analysis, like that in reference. 3 , except eight-noded quadrilateral isoparametric elements were used rather than threenoded triangular elements.

\section{Finite-Element Idealizations}

Because of the symmetries in the problem, only one quadrant of an $\mathrm{x}=$ constant plane was idealized. The quadrant $(0 \leq \mathrm{y} \leq \mathrm{b}$ and $0 \leq \mathrm{z} \leq 2 \mathrm{~h})$ is shown as the shaded region in figure $1(\mathrm{~b})$. The displacement functions $U$ and $V$ were prescribed as zero on the $y=0$ line, and the displacement function $W$ was prescribed as zero on the $z=0$ line.

The shaded region in figure $1(b)$ was idealized by eight-noded isoparametric elements in the rectangular-mesh models shown in figure 2 and the polar-mesh model in figure 3. The rectangular-mesh model had a rectangular arrangement throughout. In contrast, the polar model had a polar mesh near the free edge and rectangular arrangement elsewhere. The rectangular mesh was used to compute the stress distributions, and the polar mesh was used to investigate the stress singularities.

To study the convergence of the stresses near the free edge, three rectangular meshes were used. The medium mesh in figure $2(\mathrm{~b})$ was obtained by 
subdividing each element of the coarse mesh (fig. $2(a)$ ) into four elements. Similarly, the fine mesh in figure $2(c)$ was obtained by subdividing each element of the medium mesh into four elements. The coarse mesh had 135 nodes and 36 elements, the medium mesh had 485 nodes and 144 elements, and the fine mesh had 1833 nodes and 576 elements.

The polar mesh had the polar arrangement in the region $(19 \mathrm{~h} \leq \mathrm{y} \leq 20 \mathrm{~h}$, $0 \leq z \leq 2 h$ ) near the laminate free edge. As in the rectangular-mesh case, three meshes--coarse, medium, and fine--were used. Only the polar fine mesh is shown in figure 3. The three polar meshes had the same number of nodes and elements as their corresponding rectangular counterparts. Near the free edge, however, the polar fine mesh was an order of magnitude finer than the rectangular fine mesh.

\section{Analysis of Singularities}

The stress distribution along a radial line from a singularity can be expressed as (refs. 4 and 5)

$$
\sigma=A_{1} r^{-\alpha}+o\left(r^{-\alpha+1}\right)
$$

where $r$ is the distance from the singularity, $A_{1}$ and $\alpha$ are constants, and $0\left(r^{-\alpha+1}\right)$ represents terms of the order $r^{-\alpha+1}$ and higher. For small distances $r$; the singular term dominates and equation (3) can be approximated by

$$
\sigma \simeq A_{1} r^{-\alpha}
$$

or

$$
\log \sigma=\log A_{1}-\alpha \log r
$$


Hence, $a \log \sigma$ vs. $\log r$ plot would be a line with a slope of ${ }^{\circ}-\alpha$ and a $\sigma$-intercept of $A_{1}$. The $\alpha$ and $A_{1}$ are the power and the strength of the singularity, respectively.

Equation: (4) provided the basis for a precedure to Investigate the existence of stress singularities at the laminate edge. This equation was fitted to the computed stresses. If the equation fitted these stresses well and if the slope of the fitted line was negative, a stress singularity with power $\alpha$ was indicated. This $\log -1$ inear procedure was verified in reference 4 by applying it to several well-known singular-stress problems in twodimensional elasticity.

The log-linear procedure works best where the singular term strongly dominates the other terms. Therefore, the procedure should be applied only in the regions very close to the singularity.

\section{RESULTS AND DISCUSSION}

First, distributions are presented for interlaminar normal stress $\sigma_{2}$ and the shear stress $\sigma_{X z}$ for the various $[\theta /(\theta-90)]_{s}, 0 \leq \theta \leq 90$, laminates. Then, the results are investigated for the existence of stress singularities at the free edge. For convenience, the intersection of the interface and the free edge $(z=h ; y=b)$ will be called the interface corner. Similarly, the intersection of the midplane and the free edge $(z=0 ; y=b)$ will be called the midplane corner.

The uniform axial strain, $\varepsilon_{0}$, was arbitrarily set equal to 0.001 throughout this study.

\section{Stress Distributions}

The stresses with the steepest gradients near the free edge were the normal stress $\sigma_{z}$ and the shear stress $\sigma_{x z}{ }^{\cdot}$. The distributions for these 
stresses, through the thickness and along the interface, are presented and compared In this section.

Interlaminar normal stress, $\sigma_{z}{ }^{--}$

[0/90] laminate: Figure $4(a)$ shows the $\sigma_{z}$ distribution through the thickness along the free edge $(y=b)$ for the three rectangular meshes (coarse, medium, and fine). In this figure and all subsequent figures, the fine mesh results are represented by a curve through the data; only the value at the interface is shown as a discrete value (diamond symbol). The coarse and medium mesh results are shown by circular and square symbols, respectively. The solid symbols indicate the stresses in the top ply. As shown on the figure, the values of $\sigma_{z}$ for the three meshes agree closely except near the interface $(z=h)$. At the interface the three meshes produced noticeably different values. In addition, $\sigma_{z}$ is discontinuous across the interface; this discontinuity is slightly larger with progressive mesh refinement. Hence, the stress state shown in figure $4(\mathrm{a})$ suggests that a singularity exists at the interface corner. In contrast, at the midplane $(z=0)$ the $\sigma_{z}$ values agree for the three meshes and show no evidence of a singularity at the midplane corner.

Figure $4(\mathrm{~b})$ shows the average $\sigma_{z}$ from both plies plotted against a normalized distance from the edge. The $\sigma_{z}$ results from the three meshes are in excellent agreement for $(b-y) / h>0.08$. However, at the free edge, $y=b$, the computed $\sigma_{z}$ values are noticeably different for the three meshes, with the fine mesh producing the largest value. Therefore, results in both figures $4(\mathrm{a})$ and $4(\mathrm{~b})$ suggest that a singularity exists at the interface corner. [ \pm 45$]_{s}$ laminate: Figure $5(a)$ shows the $\sigma_{z}$ distribution through the thickness at the free edge $(y=b)$. As in the $[0 / 90]_{s}$ laminate, the values of $\sigma_{z}$ for the three meshes agree closely everywhere except near the interface $(z=h)$, where a steep gradient exists. Figure $5(b)$ shows the distribution of 
- $\sigma_{z}$ along the interface. As expected, the results from the three meshes agree except very near the free edge. Further, the computed value of $\sigma_{z}$ at the free edge was larger for progressive mesh refinement. These results for the [+45] laminate also suggest a $\sigma_{z}$ singularity at the interface corner. Other laminates: Figures 6 and 7 show the $\sigma_{2}$ distributions through the thickness and along the interface for $[15 /-75]_{s},[75 /-15]_{s},[30 /-60]_{s}$, and $[60 /-30]_{s}$, respectively. In these figures only the fine mesh results are shown. The distributions of $\sigma_{z}$ near the free edge have steep gradients for all the four laminates, indicating a possible singularity.

Comparisons: The results in figures 4 through 7 show that, except for $[0 / 90]_{s}$ and $[90 / 0]_{s}$ laminates, the interlaminar normal stress, $\sigma_{z}$, is compressive at the interface corner. In contrast, at the midplane corner only laminates $[75 /-15]_{S}$ and $[90 / 0]_{S}$ develop compressive $\sigma_{\mathbf{z}} \cdot$

Interlaminar shear stress, $\sigma_{\mathrm{xz}}{ }^{--}$

[ \pm 45$]_{S}$ laminate: Figure $8(a)$ presents the through-the-thickness distributions of $\sigma_{x z}$ at the free edge obtained using the three rectangular meshes for the $[ \pm 45]_{s}$ laminate. Figure $8(b)$ shows the $\sigma_{x z}$ distribution along the interface. For all the meshes $\sigma_{x z}$ closely agrees everywhere except near the interface in figure $8(a)$ and free edge in figure $8(\mathrm{~b})$. The gradients are steep in this region, again indicating a possible singularity for the [ ${ }^{45]_{s}}$ laminate.

other laminates: For the other laminates considered the $\sigma_{x z}$ distributions were similar to the $[ \pm 45]_{s}$ results and, therefore, are not shown. Also, the average $\sigma_{x z}$ values at the interface for a laminate and its corresponding complementary laminate (for example, $[15 /-75]_{s}$ and $[75 /-15]_{s}$ ) differed only in the third significant digit. 
Comparisons: For all laminates considered the $\sigma_{x z}$ distributions had the same sign along the interface. The [15/-75] and $[75 /-15]_{s}$ developed the largest $\sigma_{x z}$ very near the free edge compared to other laminates. Near the free edge, the $\sigma_{x z}$ stresses are larger (by at least a factor of 3 ) than the corresponding $\sigma_{z}$ stresses in each laminate, except for the $[0 / 90]_{s}$ and $[90 / 0]_{s}$ laminates for which $\sigma_{x z}$ is identically zero.

For all laminates considered, $\sigma_{x z}$ is identically zero at the midplane because of symmetry. Therefore, analysis of singularities is confined to the neighborhood of the interface corner.

\section{Singularities}

Both the $\sigma_{z}$ and $\sigma_{x z}$ distributions suggest that singularities exist at the interface corner. To identify these singularities, both $\sigma_{z}$ and $\sigma_{x z}$ stress data may be used in the log-linear procedure outlined in the analysis section. However, for the present edge-stress case (generalized plane strain), if one stress is singular at a point, then all stresses are and they all have the same singularity power, $\alpha$. This conclusion follows from the observation that these stresses have similar functional forms, derived from the same order derivatives of the same stress functions, as shown in reference 6 . Therefore, either $\sigma_{z}$ or $\sigma_{x z}$ may be used in the log-linear procedure to determine the power of the singularity.

To decide which stress was better suited for the $\log -1$ inear procedure, known singularities were examined. The case of a center-cracked plate showed that the log-linear procedure gave best results when the predominant stress was used. For the present study, the $\sigma_{x z}$ stress was predominant. As previously mentioned, the $\sigma_{x z}$ values near the edge were at least three times as large as the $\sigma_{z}$ values, except for the $[0 / 90]_{s}$ and $[90 / 0]_{s}$ laminates. Further, as 
pointed out earlier, the procedure works best when the singular term in equation (3) strongly dominates the other terms. The $\sigma_{x z}$ distributions had the same sign all along the interface; In contrast, the $\sigma_{z}$ distributions changed sign near the edge. This suggests that the singular term for $\sigma_{x z}$ is dominant over a wider range of data than for $\sigma_{2}$. For these reasons $\sigma_{x z}$ was used in the $\log -1$ inear procedure.

The results for the rectangular and polar meshes were indistinguishable for distances greater than $0.04 \mathrm{~h}$ from the free edge. Very near the free edge, however, the polar mesh yielded a greater concentration of data and, therefore, was used to analyze stress singularities.

[ \pm 45$]_{S}$ laminate. - Figure 9 shows the $\log -10 \mathrm{~g}$ plots of the absolute value of $\sigma_{x z}$, along the interface for a $[ \pm 45]_{s}$ laminate, plotted against $r$. The stresses obtained from the three polar meshes are plotted in this figure. As expected, all three meshes show a linear portion with a negative slope, indicating a singularity. Before calculating the power of the singularity, the linear region for each set of data was estimated visually. (The two points closest to the edge were neglected because they represent nodes for the element at the singularity, and this element cannot be expected to yield accurate stresses.) Stresses from the coarse, medium, and fine meshes appear to be . linear in the ranges of $0.0125 \leq r \leq 0.07,0.0035 \leq r \leq 0.015$, and $0.0015 \leq r \leq 0.01$, respectively. In these ranges a linear least-squarefitting procedure was used to determine the power $(\alpha)$ and the strength $\left(A_{1}\right)$ of the singularity in the equation

$$
\log \sigma_{x z}=\log A_{1}-\alpha \log r
$$

The following values were found for the three meshes. 


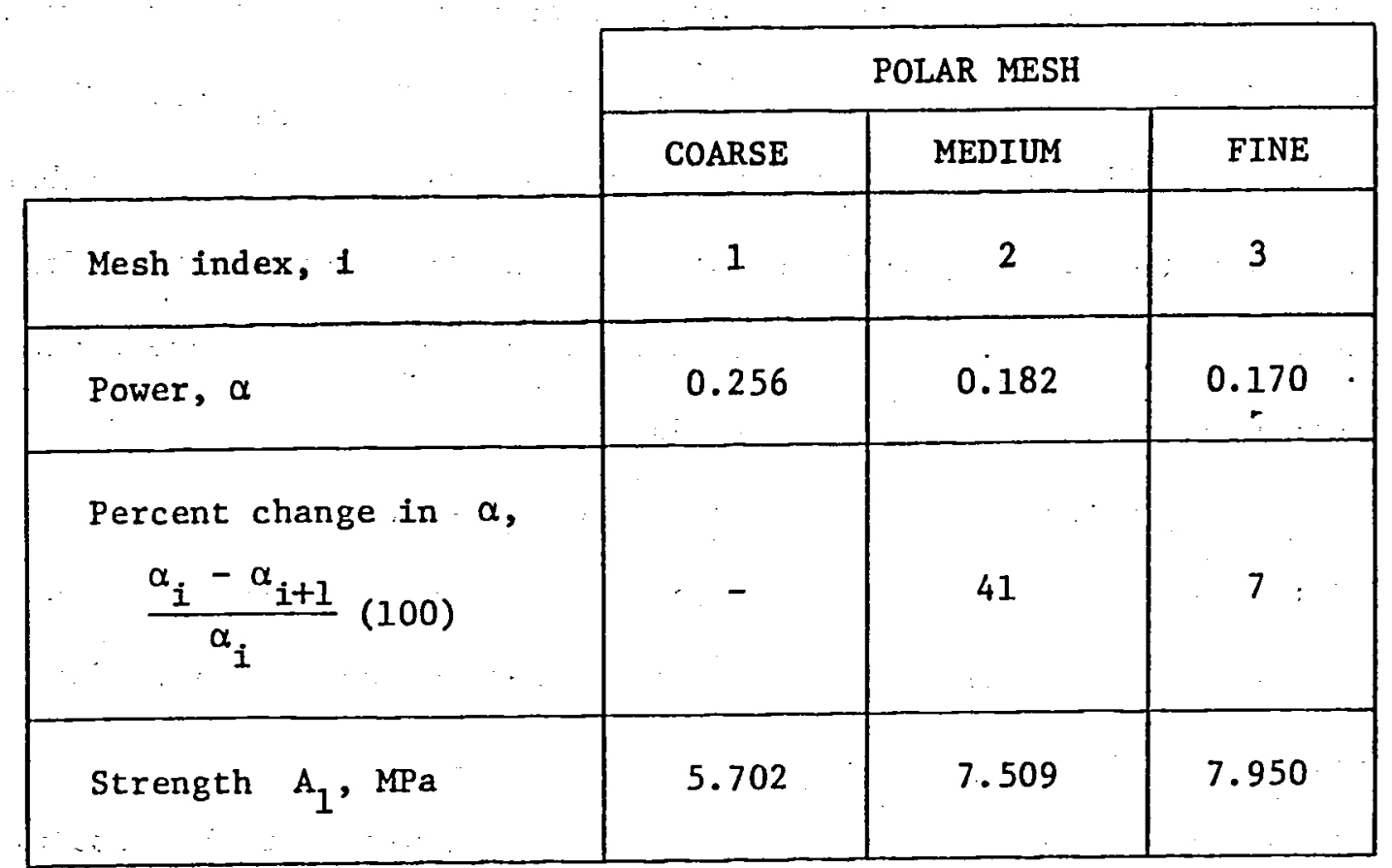

As shown in the table, by refining the coarse mesh to generate the medium "mesh, the $\alpha$ changed by 41 percent. For the fine mesh, the additional refinement produced only a 7-percent change, which is only about one-sixth the 42-percent change. Further mesh refinement (dividing each fine mesh element into four elements) would probably cause an additional 1-percent change (one-sixth of 7 percent). This 1-percent change is neglibibly small. Similar arguments can be made for the strength of the singularity, $A_{1}$. Therefore, the present fine mesh results were treated as converged values.

To check the accuracy of the present procedure, the $[ \pm 45]_{s}$ singularity was computed using the $\sigma_{\mathrm{xz}}$ stresses along nine different radial lines of the polar mesh. - The calculated power of the singularity $\alpha$ should be the same for all such lines. Figure 10 shows $\alpha$ and the corresponding $A_{1}$ values plotted against $\phi$, the angular position of each radial line. These values of $\alpha$ vary slightly, from 0.195 at $\phi=0$ to an interface value of 0.170 at $\phi=\pi / 2$. At the interface, the $\sigma_{x z}$ stresses were largest, as shown by the 
largest $A_{1}$ value at $\phi=\pi / 2$ in figure 10 . As mentioned earlier, the present procedure yields best results when the predominant stresses are used. Because the $\sigma_{x z}$ stress was largest at the interface $(\phi=\pi / 2)$, the $\alpha$ value of 0.170 is believed to be accurate. Also, the absolute value of the correlation coefficient in the least-square procedure was closest to unity (0.9996) for $\phi=\pi / 2$.

Other laminates. - The $\sigma_{x z}$ stress was also used to identify the singularities for the $[15 /-75]_{s},[75 /-15]_{s},[30 /-60]_{s}$, and $[60 /-30]_{s}$ laminates. Figure 11 shows the $\log -\log$ plots for these four laminates. For comparison the previous results for the [ \pm 45$]_{s}$ laminate are also included in this figure. As pointed out earlier, for a laminate and its complementary laminate the $\sigma_{x z}$ results differed only in the third significant digit. Therefore, the power of the singularity for a laminate and its complement is apparently identical. Also note that log-linear plots in figure 11 are parallel to each other. Therefore, the power of the singularities for [15/-75] ${ }^{\prime}[75 /-15] \mathrm{s}$ ' $[30 /-60]_{s}$, and $[60 /-30]_{s}$ are identical to that for the $[ \pm 45]_{s}$ case, that is, 0.170. However, the three parallel lines in figure 11 are separated, indicating different singularity strengths, $A_{1}$.

Figure 12 shows $\alpha$ and $A_{1}$ for various $[\theta /(\theta-90)]_{s}$ laminates as functions of the outer ply angle, $\theta$. To investigate $\alpha$ and $A_{1}$ in the region $0 \leq \theta \leq 15$ (and $75 \leq \theta \leq 90$ ), the laminates $[2 /-88]_{s}$ and $[7.5 /-82.5]_{s}$ were also considered. The $\alpha$ values for these laminates are nearly equal to 0.170 , but the $A_{1}$ values are much smaller than for the other laminates. This was expected because the $\sigma_{\mathrm{xz}}$ stress is identically zero for $[0 / 90]_{s}$ and $[90 / 0]_{s}$ laminates; thus, for $\theta$ nearly equal to zero $A_{1}$ should also be very small. Dotted lines in figure 12 represent extrapolations to zero. 
- Figure 12 shows that both $\alpha$ and $A_{1}$ are symmetric about $\theta=45^{\circ}$. Also, the strength, $A_{1}$, is maximum for the $[15 /-75]_{s}$ and $[75 /-15]_{s}$ laminates. $[0 / 90]_{s}$ and $[90 / 0]_{s}$ laminates.- For the $[0 / 90]_{s}$ laminate, $\sigma_{z}$ was used

-in the log-linear procedure because the $\sigma_{x z}$ was identically zero. As expected, the log-log plot in figure 13 shows a linear region with a negative slope. The power of the singularity was obtained from the least-square procedure as 0.202 . This power is larger than the 0.17 calculated from other laminates but is only slightly beyond the range shown in figure 10 . The computed power of 0.202 probably occurred because the $\sigma_{z}$ singulartty for the $[0 / 90]_{s}$ laminate was not as dominant as the $\sigma_{x z}$ singularity analyzed for the other laminates. However, if the log-linear procedure were applied to $\sigma_{z}$ stresses for smaller $r$ values, the power may a] so approach 0.17 . Such calculations for smaller $r$ values would require further mesh refinement, which is beyond the scope of this paper.

For the $[90 / 0]_{s}$ laminate neither $\sigma_{z}$ nor $\sigma_{x z}$ could be used in the log-linear procedure. The $\sigma_{\mathrm{xz}}$ was identically zero and the $\sigma_{\mathrm{z}}$ changed sign very near the free edge (see fig. $4(\mathrm{~b})$ ). Consequently, the singularity for the $[90 / 0]_{s}$ laminate was not computed. However, as shown previously for other laminates, a laminate and its complementary laminate have the same $\alpha$. . Therefore, by induction, the singularities for $[0 / 90]_{\mathrm{S}}$ and $[90 / 0]_{\mathrm{S}}$ should also be identical and $\alpha$ should be 0.17 .

In general, these results suggest that the power of the singularities for $[\theta /(\theta-90)]_{s}, \quad 0 \leq \theta \leq 90$, laminates with material properties used in this paper is about 0.17 . This power should be interpreted only as an approximate value because of the numerical accuracy of the $\log -1$ inear procedure. 
CONCLUDING REMARKS

The stresses near a straight, free edge in composite laminates subjected to uniform axial strain were studied by a quasi-three-dimensional finiteelement analysis. Seven different four-ply laminates were analyzed: [0/90] ${ }_{s}$.

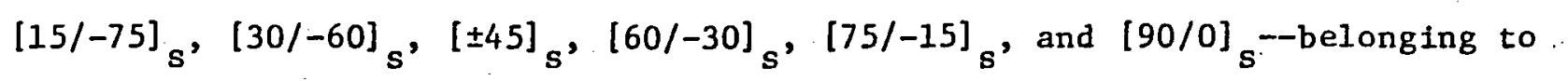
the family $[\theta /(\theta-90)]$, $0 \leq \theta \leq 90$. The laminates were 1dealized by eightnoded isoparametric elements. Systematic convergence studies were made with the aid of three meshes to examine the interlaminar stresses near the free edge.

Near the intersection of the interface and the free edge, the interlaminar normal stress $\sigma_{z}$ was tensile for $[0 / 90]_{s}$ and $[90 / 0]_{s} 1$ laminates and was compressive for all other laminates considered. The interlaminar shear stress $\sigma_{x z}$. was much larger than the normal stress $\sigma_{z}$ for all laminates except for $[0 / 90]_{s}$ and $[90 / 0]_{s}$ laminates (for which $\sigma_{x z}$ is identically zero). Among the laminates considered, laminates $[15 /-75]_{s}$ and $[75 /-15]_{s}$ developed the highest $\sigma_{\mathrm{xz}}$.

Convergence studies indicated that stress singularities probably exist at the intersection of the interface and the free edge, and the singularities do not exist at the midplane. A log-linear curve fitting procedure appears to . confirm the existence of a singular point at the intersection of the interface and free edge. The shear stress data close to the singular point were used in the log-linear procedure to evaluate the power of the singularities.

The present results suggest that the power of the singularity is the same for all orthotropic laminates in the family represented by $[\theta /(\theta-90)]_{\mathrm{s}}, 0 \leq \theta \leq 90$ for a given set of material properties. For these laminates, with material properties of the graphite/epoxy considered, the power of the singularity was about 0.17 . 


\section{REFERENCES}

1. R. B. Pipes and N. J. Pagano, Interlaminar stresses in composite laminates under uniform axial extension. J. Comp. Materials 4, 538-548 (1970).

2. E. F. Rybicki, Approximate three-dimensional solutions for symmetric laminates under in-plane loading. J. Comp. Materials 5, 354-360 (1971).

3. A. S. D. Wang and F. W. Crossman, Some new results on edge effect in symmetric composite laminates. J. Comp. Materials 11, 92-106 (1977).

4. E. B. Becker, R. S. Dunham, and M. Stern, Some stress intensity calculations using fintte elements. Proceedings of the 1974 International Conference on Finite Element Methods in Engineering (Edited by V. A. Pulmans and A. P. Kabaila, 117-138 (1974).

5. M. L. Williams, Stress singularities resulting from various boundary conditions in angular corners of plates in extension. J. Appl. Mech. 19, 526-528 (1952).

6. S. G. Lekhnitskii, Theory of Elasticity of an Anisotropic Elastic Body. Holden-Day, Inc., San Francisco (1963). 


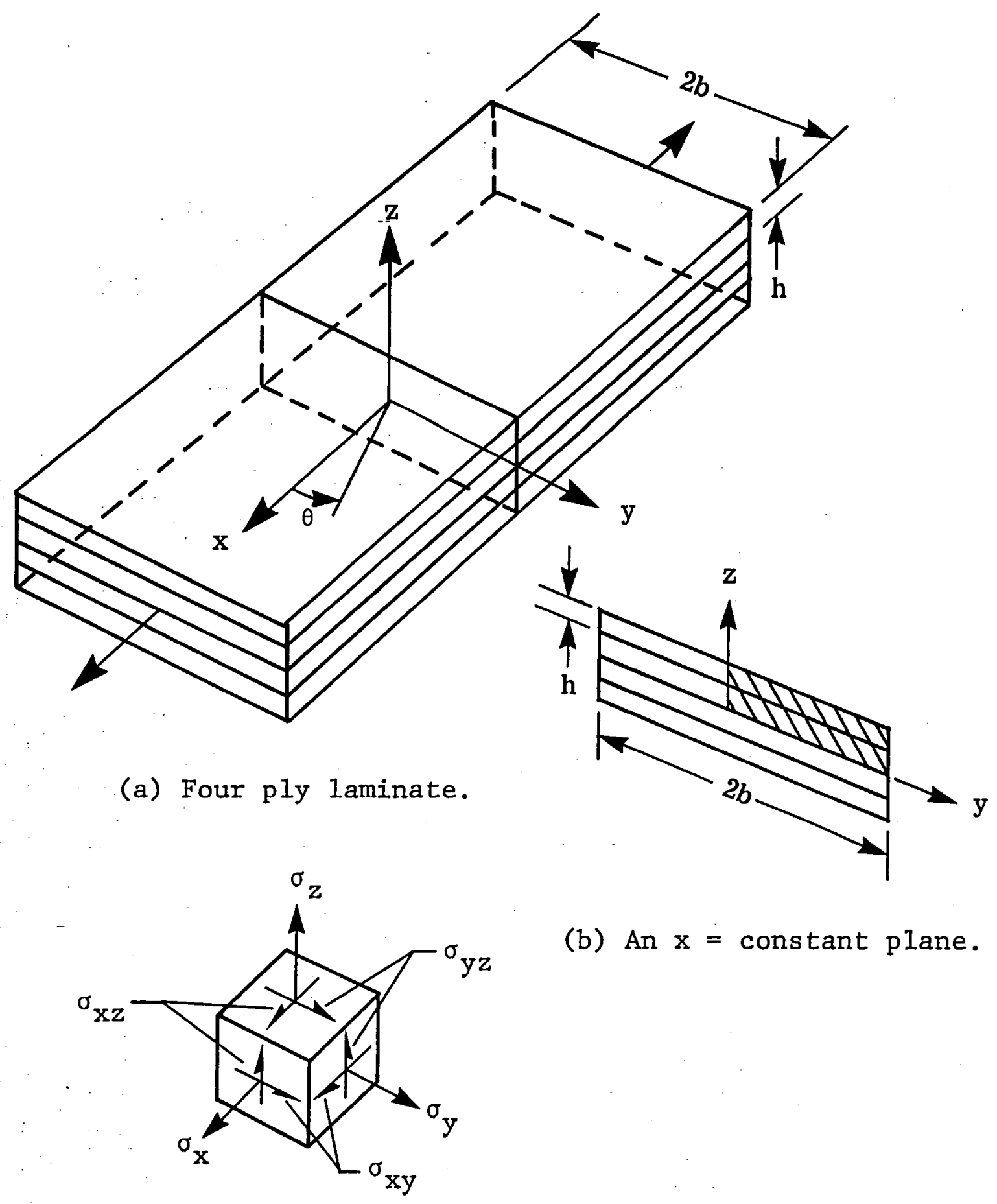

(c) 3-D stress components.

Figure 1.- Laminate configuration, loading, and stresses. 


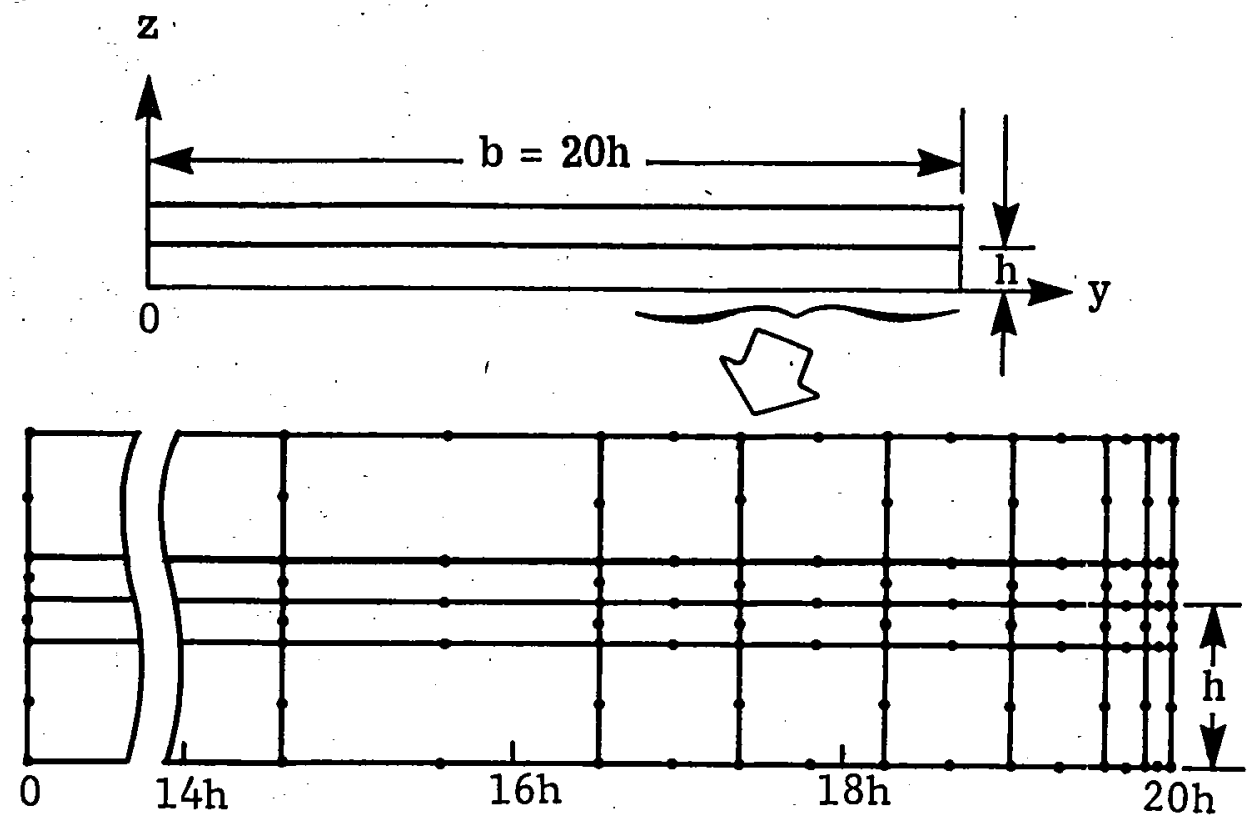

(a) Coarse mesh.

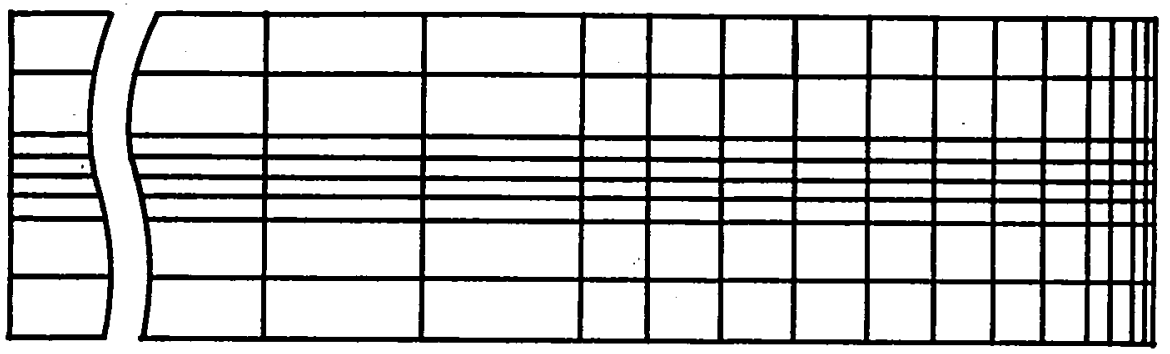

(b) Medium mesh.

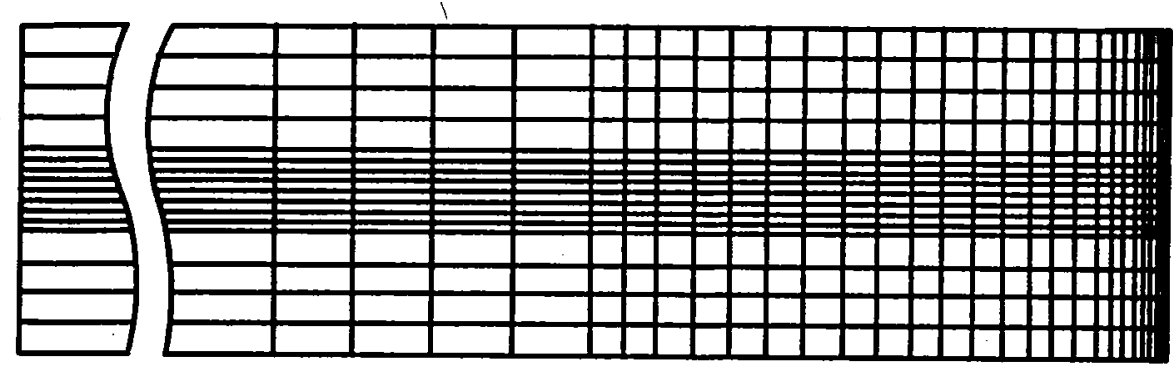

(c) Fine mesh.

Figure 2.- Rectangular mesh models. 


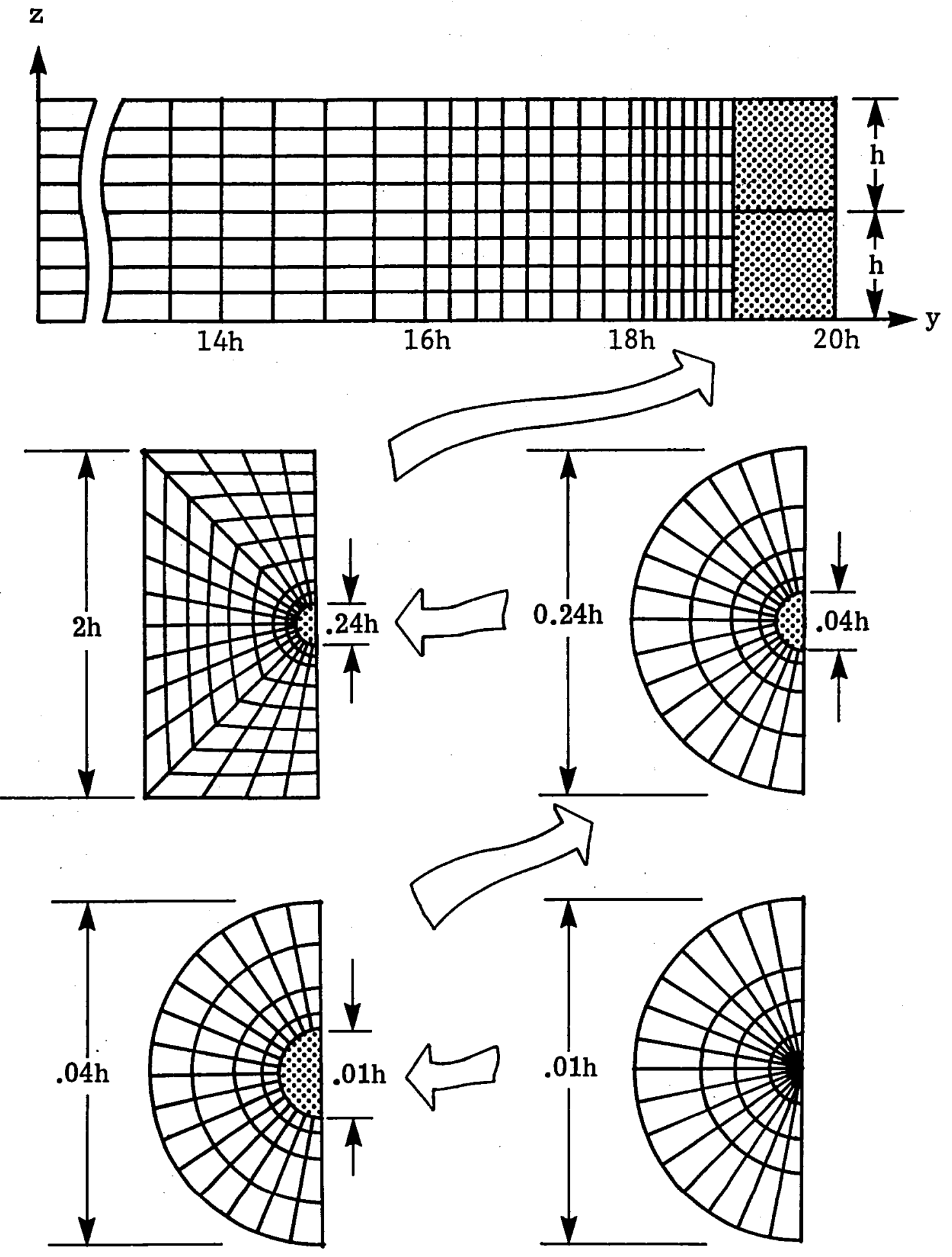

Figure 3.- Fine polar mesh model. 


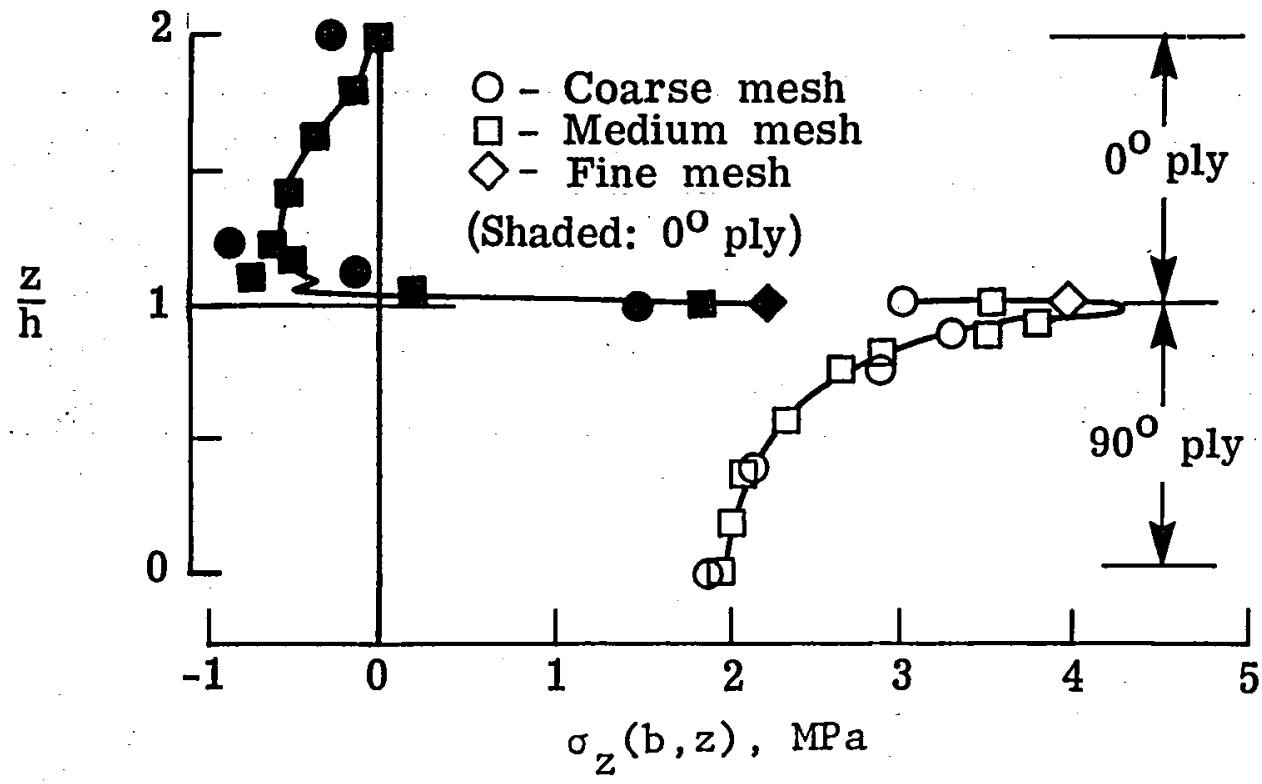

(a) $\sigma_{z}$ along the free edge, $y=b$.

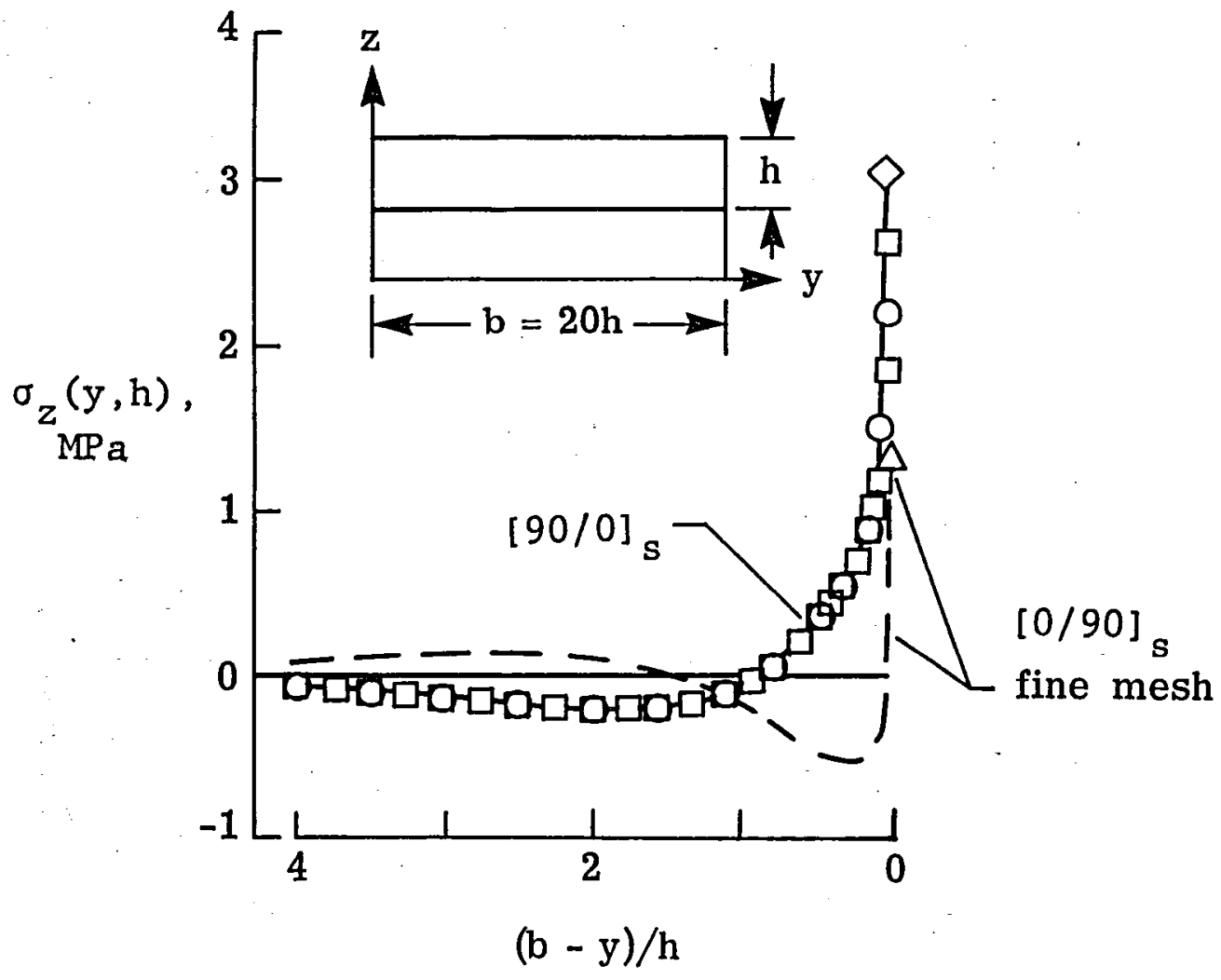

(b) $\sigma_{z}$ along the interface, $z=h$.

Figure 4.- The $\sigma_{z}$ distributions for $[0 / 90]_{s}$ and $[90 / 0]_{s}$ laminates. 


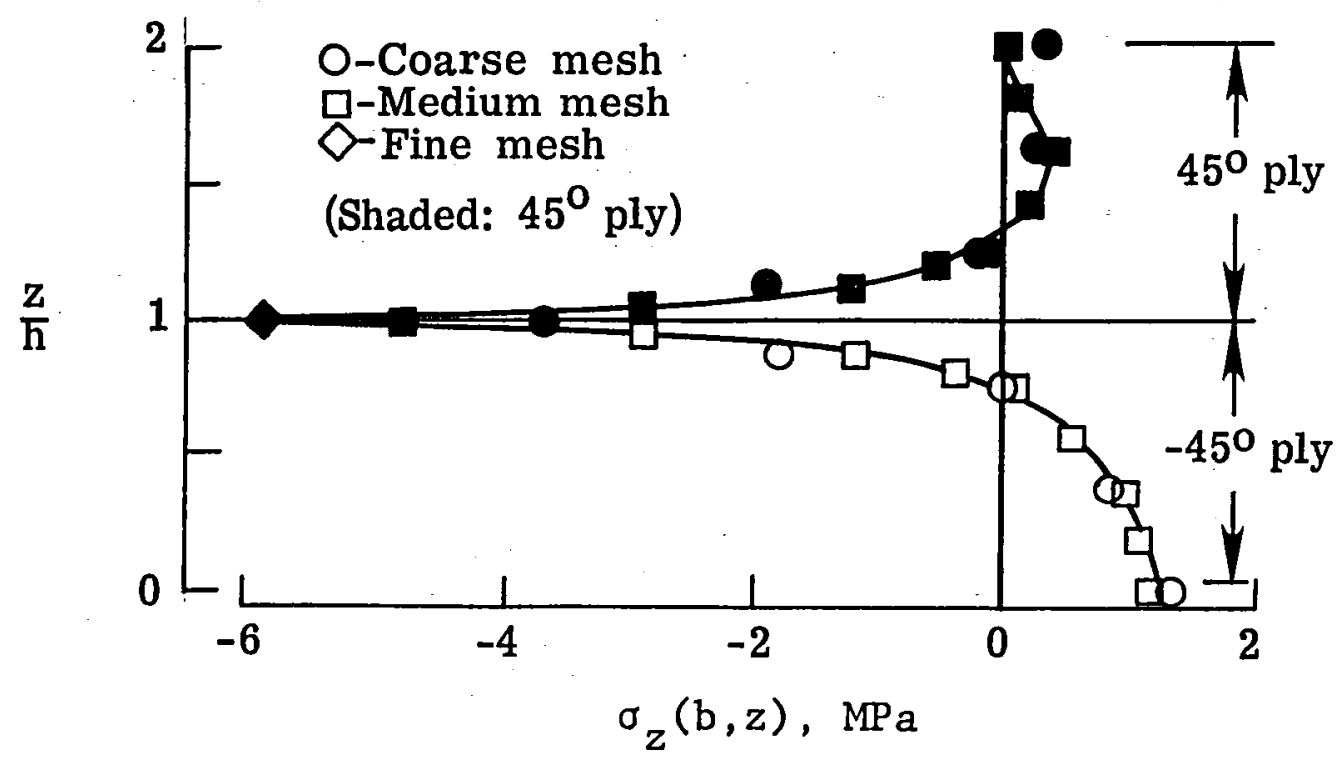

(a) $\sigma_{z}$ along the free edge, $y=b$.

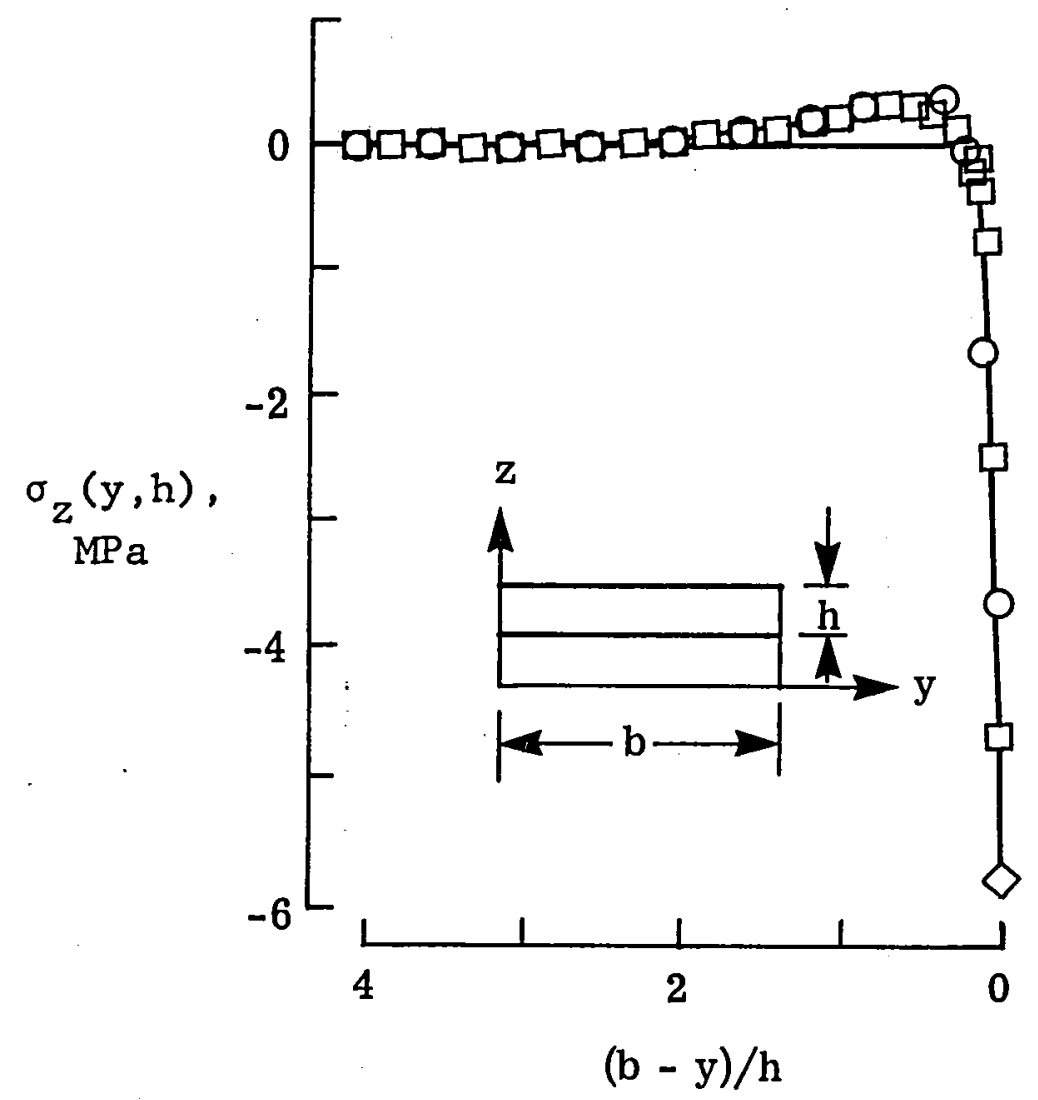

(b) $\sigma_{z}$ along the interface, $z=h$.

Figure 5.- The $\sigma_{z}$ distributions for $[ \pm 45]_{s}$ laminate. 


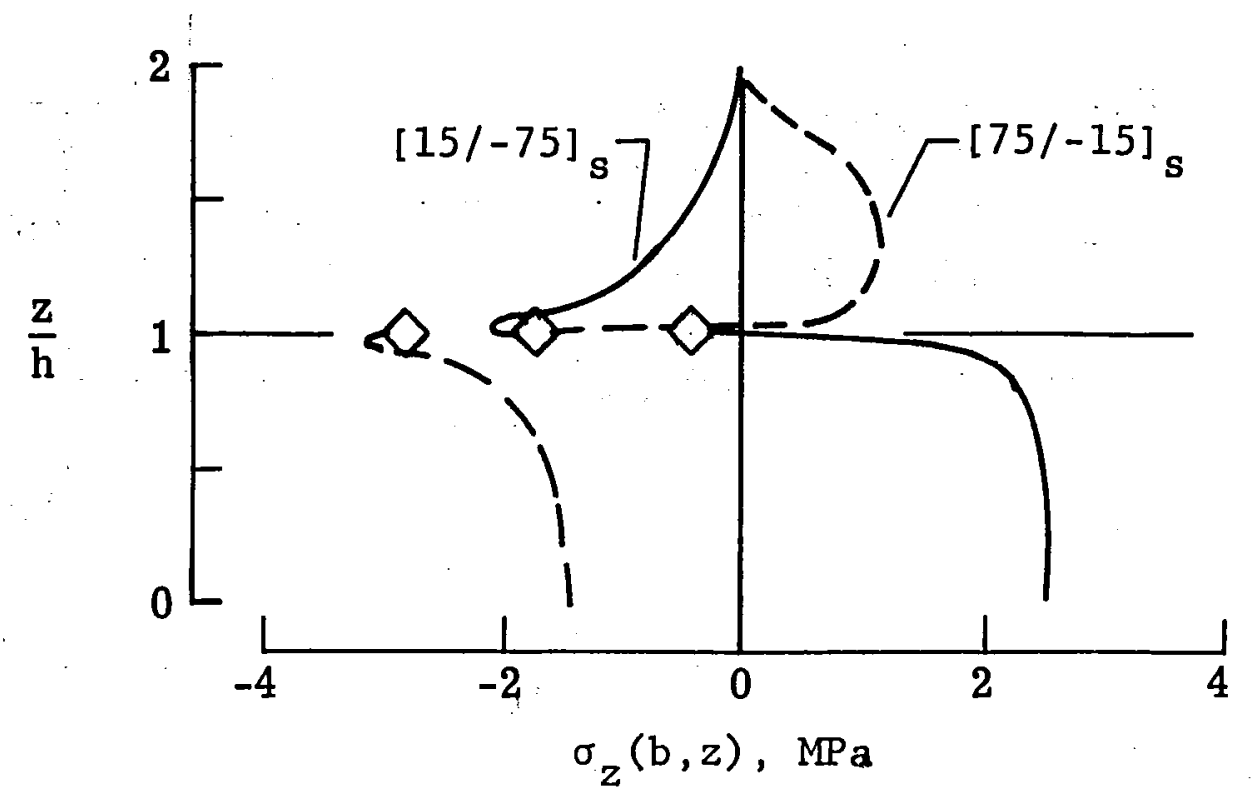

(a) $\sigma_{z}$ along the free edge, $y=b$.

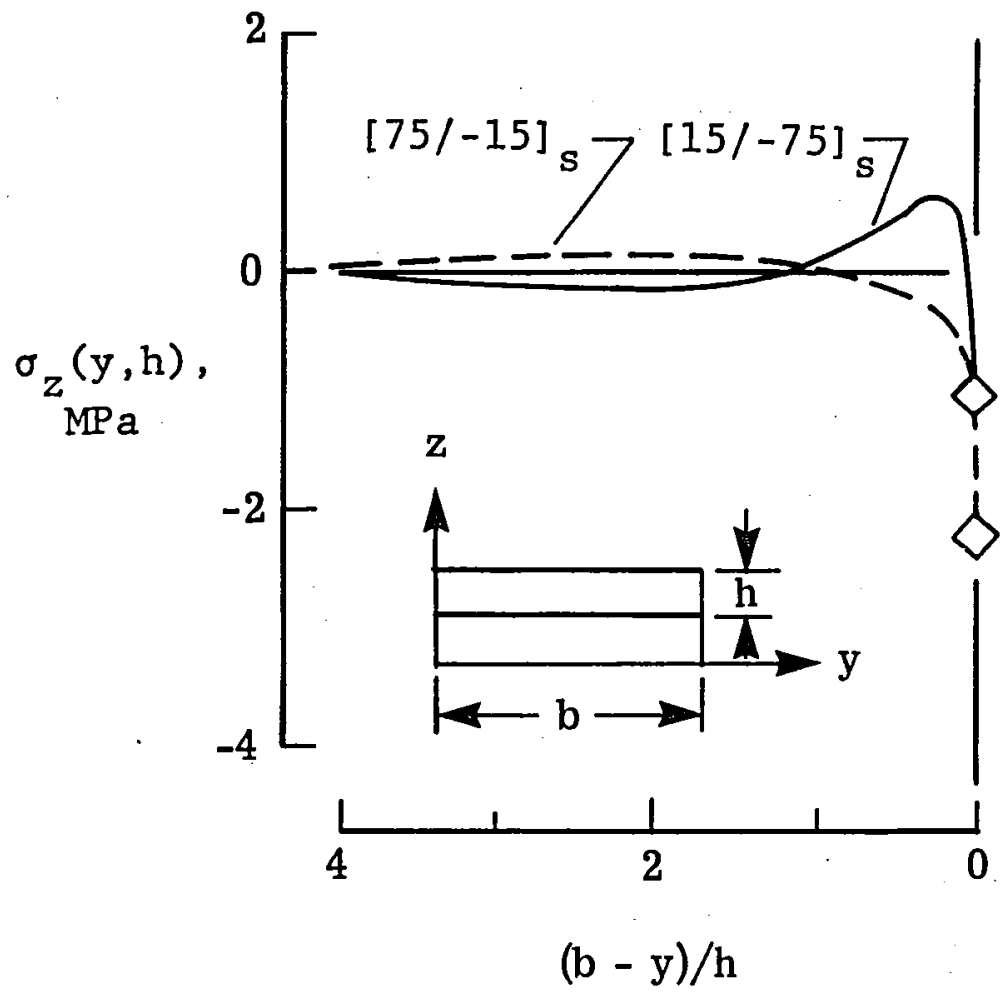

(b) $\sigma_{z}$ along the interface, $z=h$.

Figure 6.- The $\sigma_{z}$ distributions for $[15 /-75]_{s}$ and $[75 /-15]_{s}$ laminates, fine mesh. 


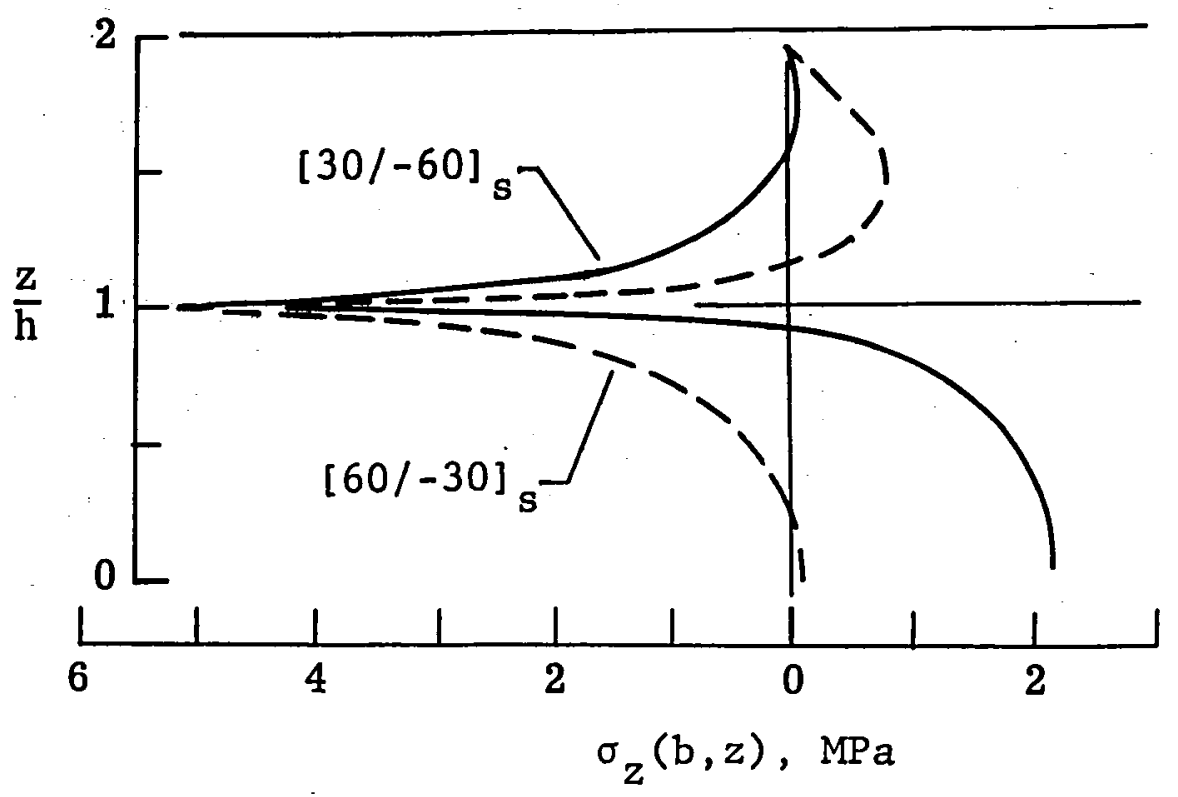

(a) $\sigma_{z}$ along the free edge, $y=b$.

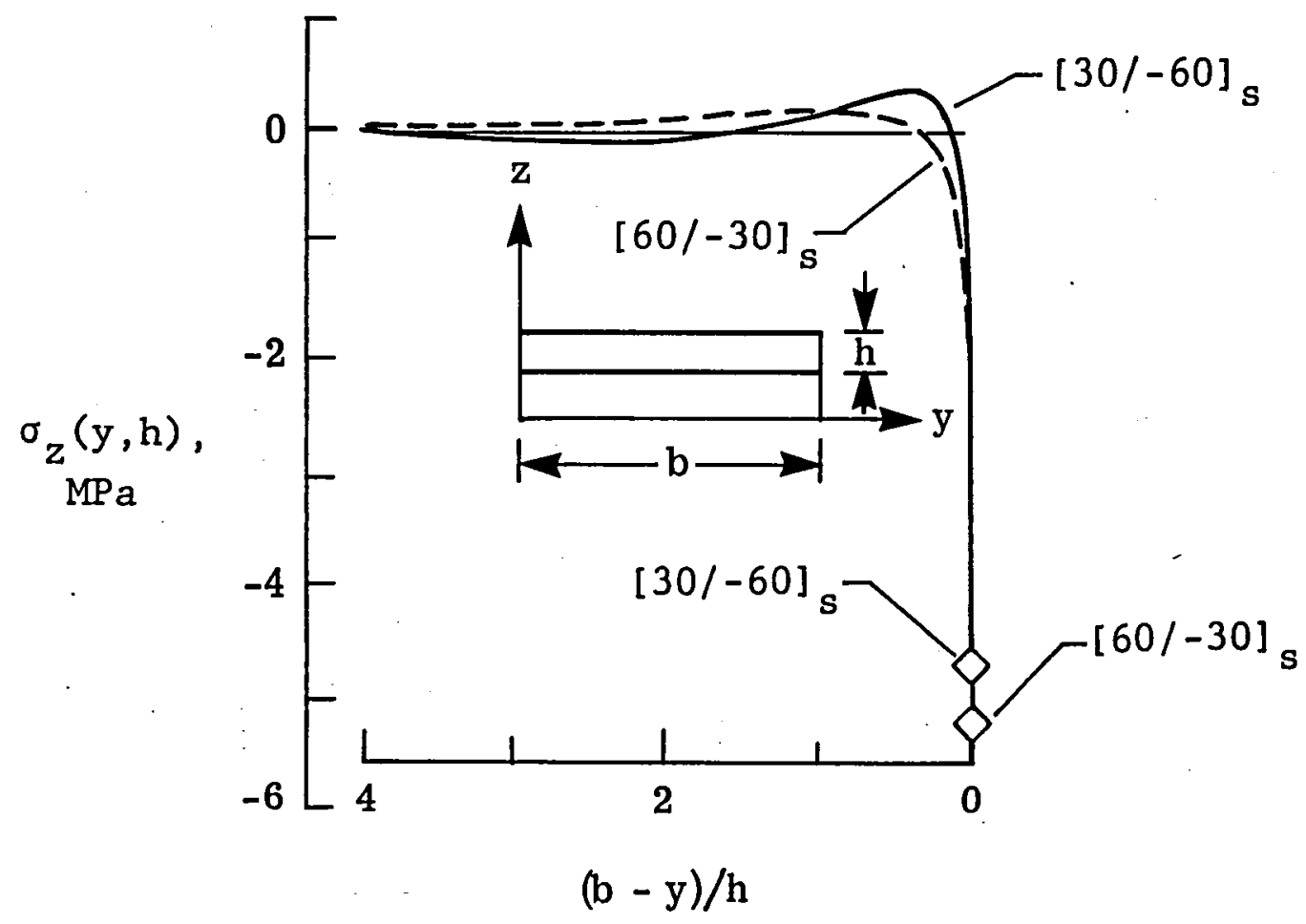

(b) $\sigma_{z}$ along the interface, $z=h$.

Figure 7.- The $\sigma_{z}$ distributions for $[30 /-60]_{s}$ and $[60 /-30]_{s}$ laminates, fine mesh. 


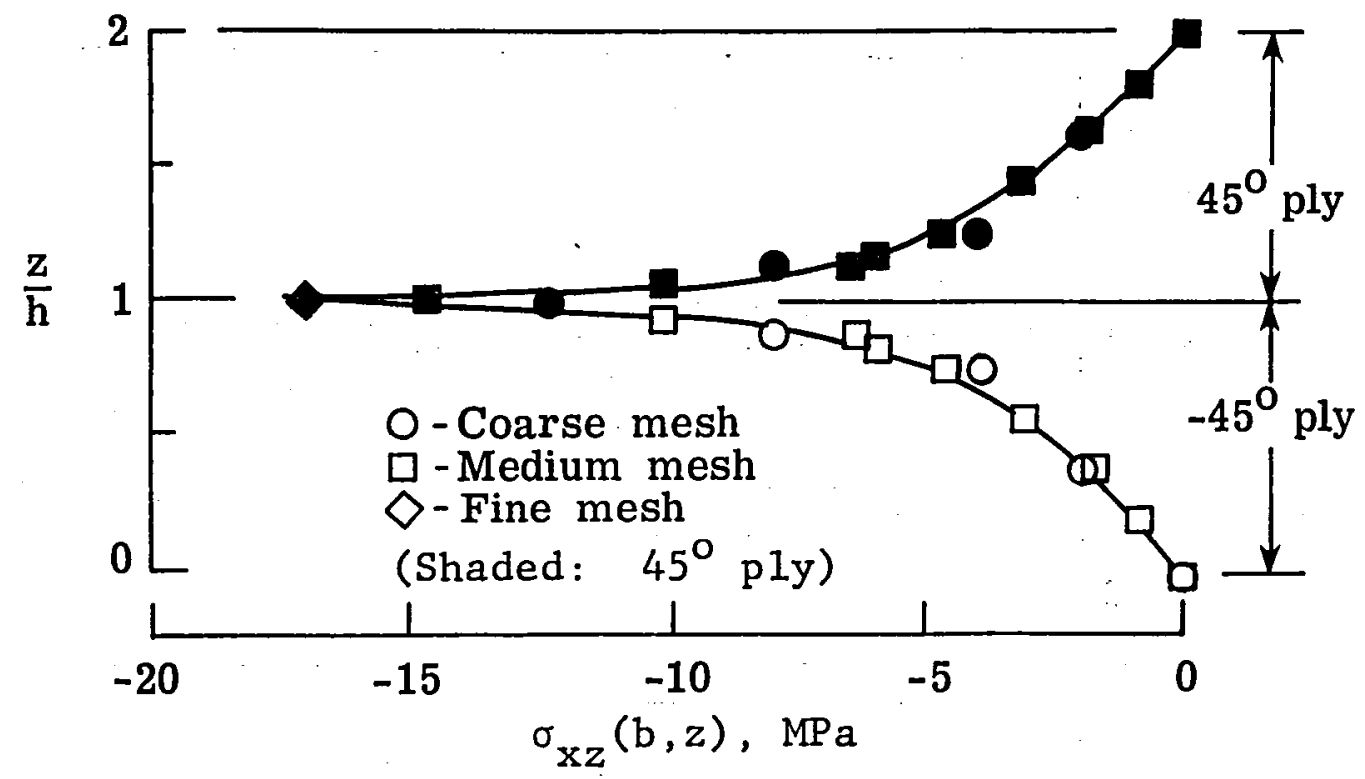

(a) $\sigma_{x z}$ along the free edge, $y=b$.

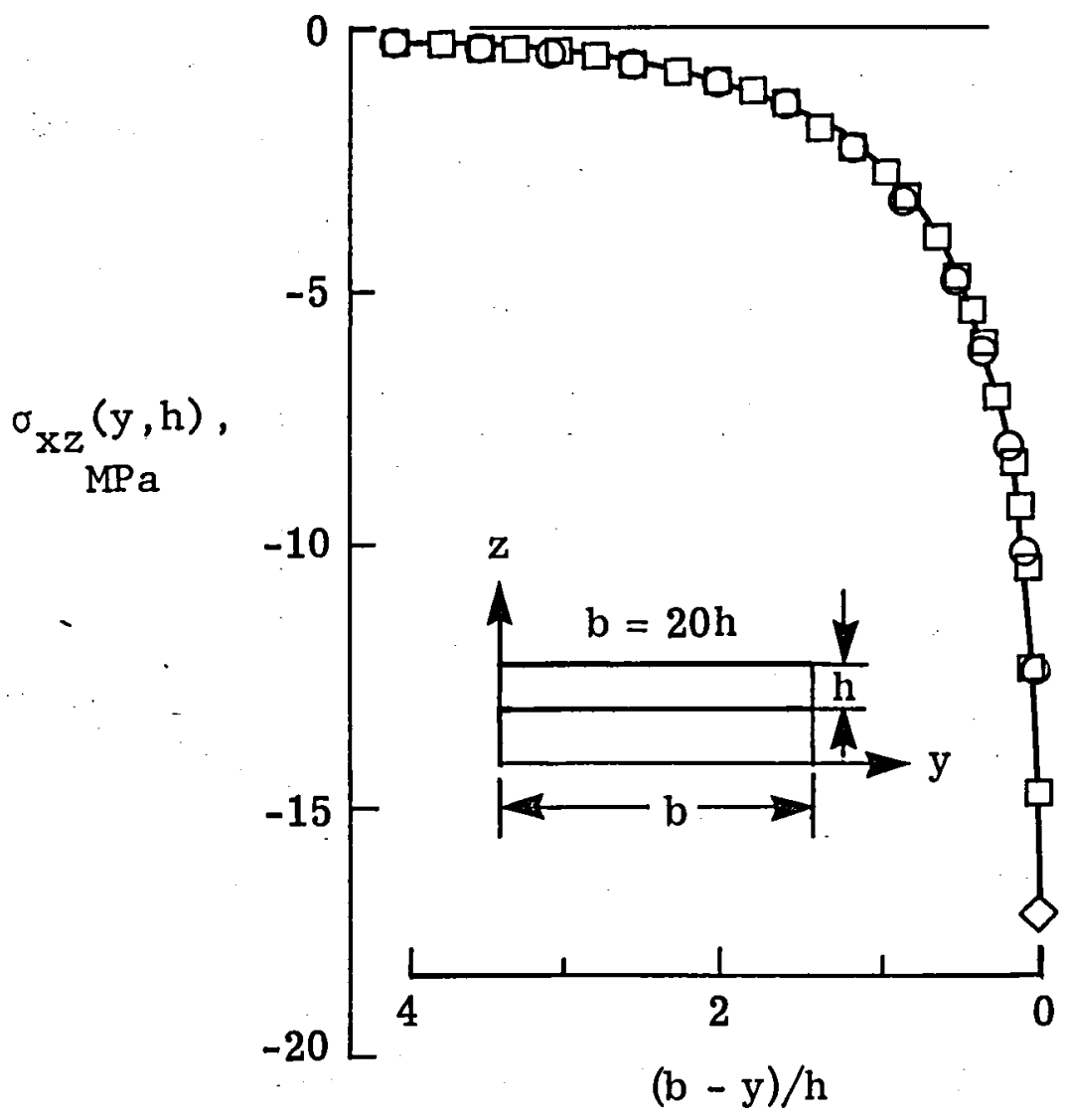

(b) $\sigma_{x z}$ along the interface, $z=h$.

Figure 8. - The $\sigma_{\mathrm{xz}}$ distributions for the $[ \pm 45]$ s laminate. 


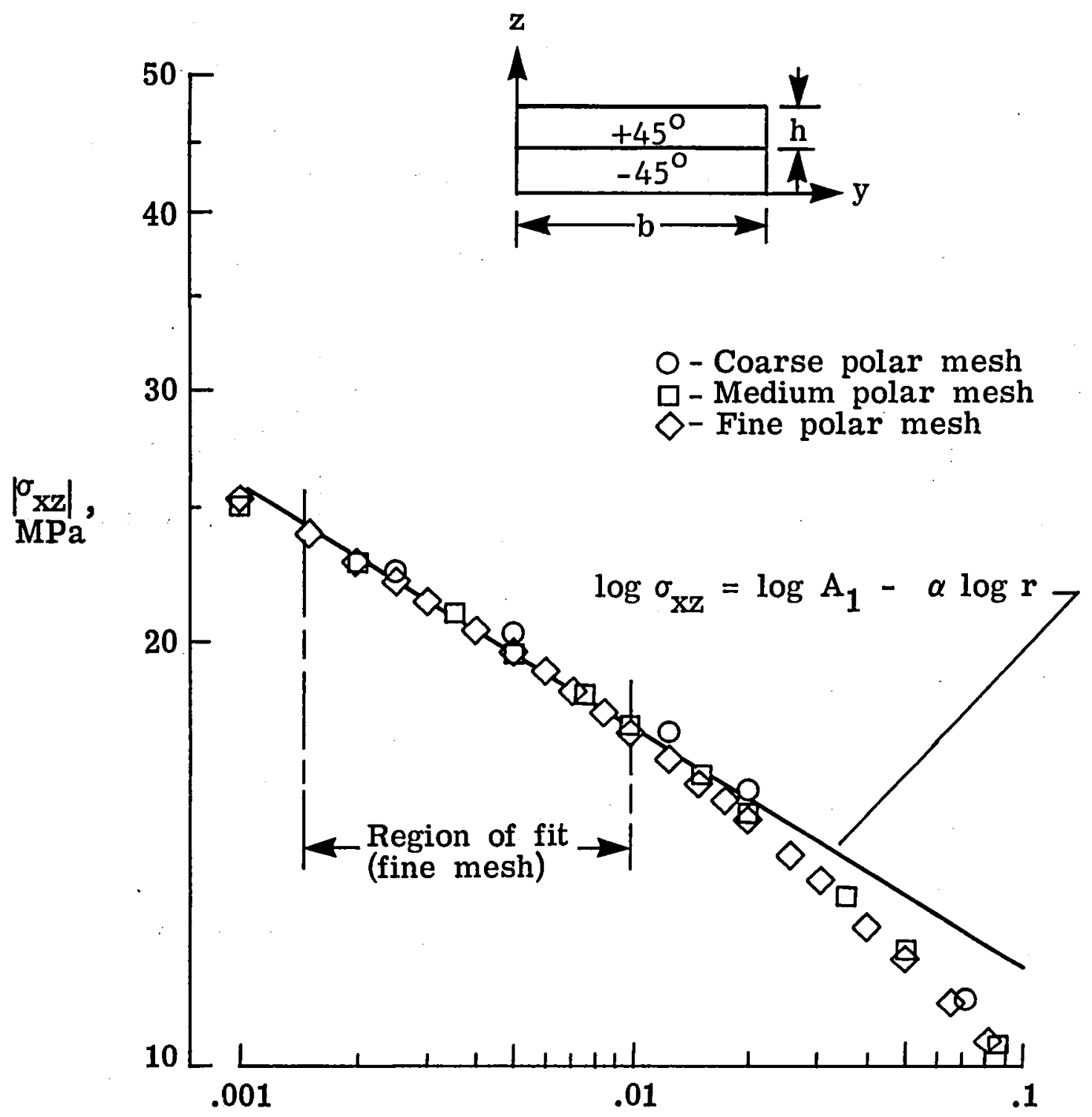

Normalized distance from the edge, $r$

Figure 9.- Log-log plot for $\sigma_{x z}$ along the interface $(z=h)$ for the $[ \pm 45]_{s}$ laminate. 


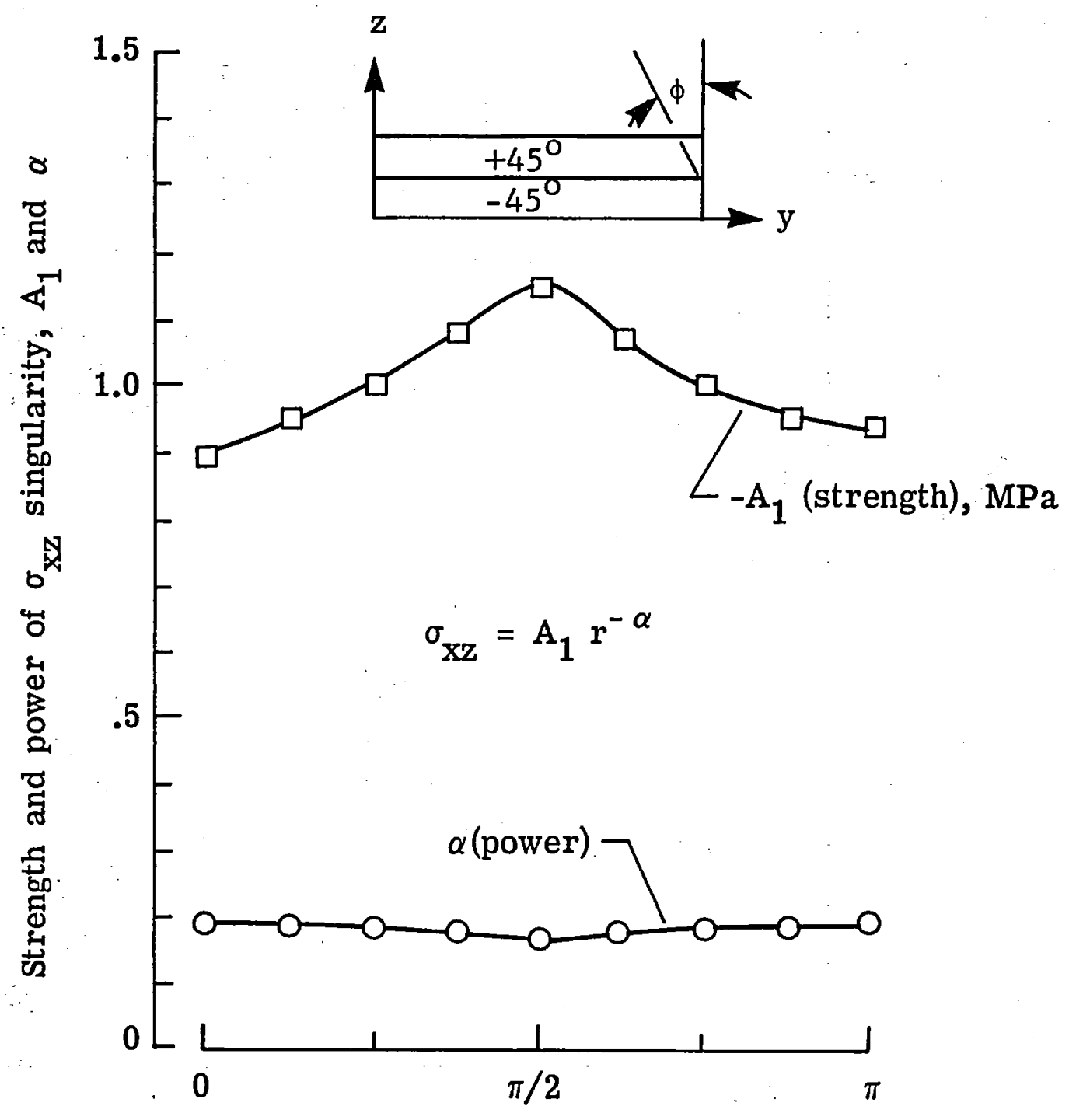

Angle, $\phi$

Figure 10.- Angular variation of strength and power of $\sigma_{x z}$ singularity for $[ \pm 45]_{s}$ laminate. 


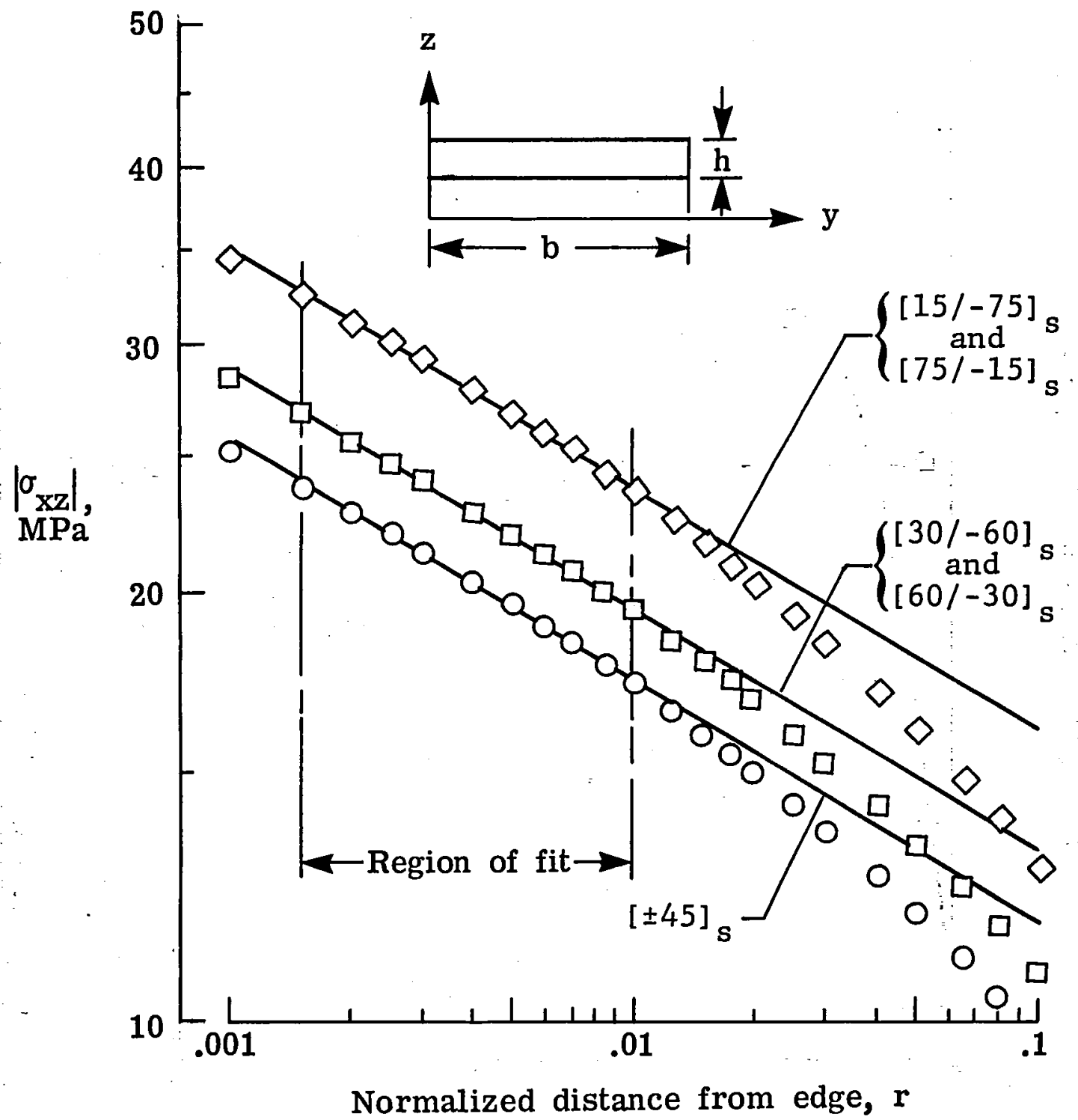

-Figure 11.- Log-log plot for $\sigma_{x z}$ along interface $(z=h)$ for various laminates. 




Figure 12. - Strength and power of $\sigma_{\mathrm{xz}}$ singularity for $[\theta /(\theta-90)]_{\mathrm{S}}$, $0 \leq \theta \leq 90$, laminates. 


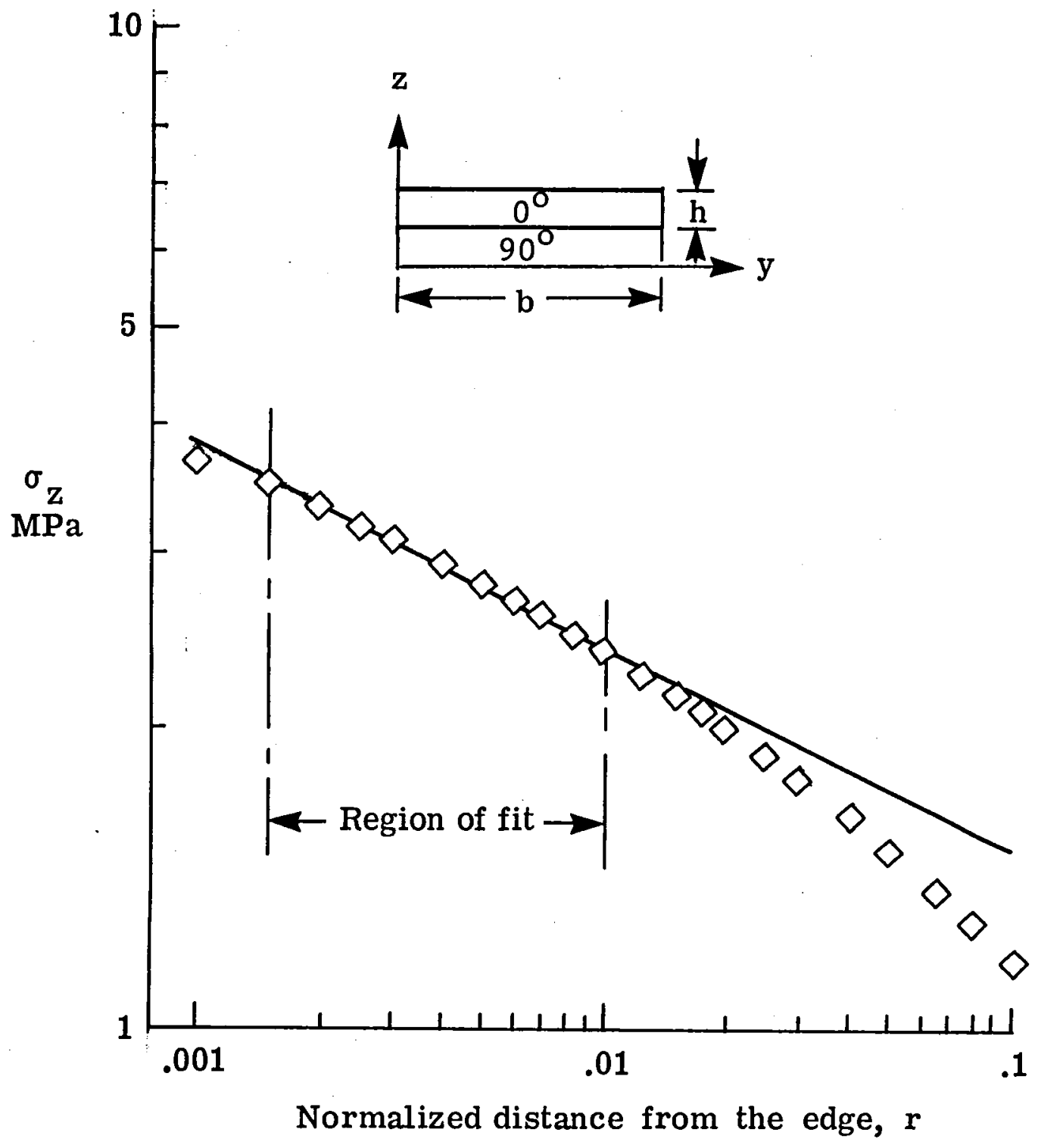

Figure 13.- Log-log plot for $\sigma_{z}$ along the interface $(z=h)$ for a $[0 / 90]$ s laminate. 

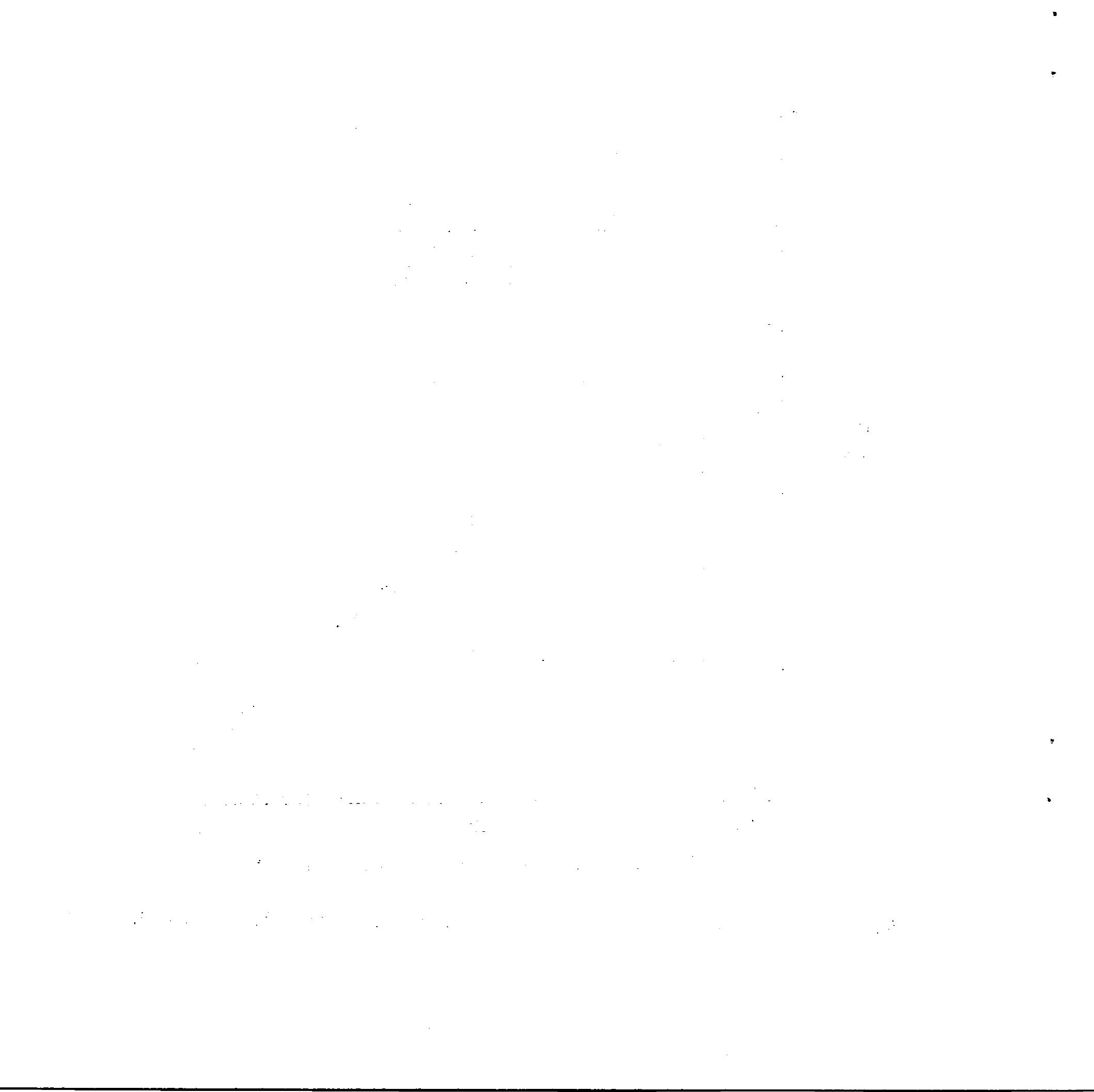


\begin{tabular}{|c|c|}
\hline $\begin{array}{l}\text { 1. Report No. } \\
\text { NASA TM- } 81876\end{array}$ & 3. Recipient's Catalog No. \\
\hline \multirow{2}{*}{$\begin{array}{l}\text { 4. Title and Subtitle } \\
\text { INTERLAMINAR STRESS SINGULARITIES AT A STRAIGHT FREE EDGE } \\
\text { IN COMPOSITE LAMINATES }\end{array}$} & $\begin{array}{l}\text { 5. Report Date } \\
\text { August } 1980\end{array}$ \\
\hline & 6. Performing Organization Code \\
\hline $\begin{array}{l}\text { 7. Author(s) } \\
\text { I. S. Raju and John H. Crews, Jr. }\end{array}$ & 8. Performing Organization Report No. \\
\hline \multirow{2}{*}{$\begin{array}{l}\text { 9. Performing Organization Name and Address } \\
\text { NASA Langley Research Center } \\
\text { Hampton, VA } 23665\end{array}$} & $506-53-53-01$ \\
\hline & 11. Contract or Grant No. \\
\hline \multirow{2}{*}{$\begin{array}{l}\text { 12. Sponsoring Agency Name and Address } \\
\text { National Aeronautics and Space Administration } \\
\text { Washington, DC } 20546\end{array}$} & $\begin{array}{l}\text { 13. Type of Report and Period Covered } \\
\text { Technical Memorandum }\end{array}$ \\
\hline & 14. Sponsoring Agency Code \\
\hline
\end{tabular}

15. Supplementary Notes

16. Abstract

A quasi-three-dimensional finite-element analysis was used to analyze the edgestress problem in four-ply, composite laminates. The seven laminates that were considered belong to the laminate family $[\theta /(\theta-90)]_{S}$, where $0 \leq \theta \leq 90$. Systematic convergence studies were made to explore the existence of stress singularities near the free edge. The present analysis appears to confirm the existence of stress singularities at the intersection of the interface and the free edge. The power of the stress singularity was the same for all seven laminates considered.

17. Key Words (Suggested by Author(s))

Composite laminates

Interlaminar stresses

Finite elements

Stress singularity
18. Distribution Statement

$$
\text { Unclassified - Unlimited }
$$

Subject Category 39
19. Security Classif. (of this report) Unclassified
20. Security Classif. (of this page)

Unclassified
21. No. of Pages

29
22. Price"

A03

* For sale by the National Technical Information Service, Springfield, Virginia 22161 



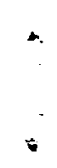

$+$ 